\title{
Searches for Dark Matter with in Events with Hadronic Activity
}

\section{Gabriele Chiodini, On behalf of the ATLAS Collaboration.}

INFN sezione di Lecce, via Arnesano 73100 Lecce - Italy

\begin{abstract}
The astrophysical evidence of dark matter provides some of the most compelling clues to the nature of physics beyond the Standard Model. From these clues, ATLAS has developed a broad and systematic search program for dark matter production in LHC collisions.

In the framework of Simplified models the searches are divided into invisible and visible channels, corresponding to dark matter searches, with a missing energy signature, and dark matter mediator searches, looking for bump in invariant mass distributions.

This report illustrates these searches in events with hadronic activity in the most representative channel for each of the two main strategies: mono-jet plus missing energy and di-jet events. The data were recorder by the ATLAS experiment in proton-proton collisions at the LHC with center of mass energy of $13 \mathrm{TeV}$ in 2015 and 2016, corresponding to an integrated luminosity of 36.1 $\mathrm{fb}^{-1}$, for mono-jet plus missing energy events, and $37 \mathrm{fb}^{-1}$, for di-jet events.
\end{abstract}

EPS-HEP 2017, European Physical Society conference on High Energy Physics 5-12 July 2017

Venice, Italy 


\section{Introduction}

Dark matter (DM) evidences are a big challenge for any explanation other than the existence of some new fundamental particle, that remains to be discovered [2]. Modified gravity theories [3], for example, are unable to account for the bullet cluster dynamics. In this respect, excellent candidates are the so called WIMPs: stable, neutral, color-singlet and weakly interacting particles. WIMPs lead to the correct dark matter relic density for a mass between a few $\mathrm{GeV}$ and one $\mathrm{TeV}$ and cross sections of the order of electroweak interactions (WIMPs miracle), and, intriguing enough, in this case they should be related to the gauge hierarchy problem.

If WIMPs exist, they are likely going to be produced at LHC in pairs. Thus, a significant imbalance in the transverse momentum ( $\mathrm{E}_{T}^{\text {miss }}$ ) of the event is possible only if a visible standard model (SM) particle recoils against them, typically through initial state radiation (ISR). The searches $\mathrm{pp} \rightarrow \chi \bar{\chi}+\mathrm{X}$ are the most model independent and are referred to as mono-X+MET searches, where $\mathrm{X}$ can be a jet, a W/Z boson, a Higgs or a heavy quark pair, such as $t \bar{t}$ and $b \bar{b}$.

In Simplified models the DM mediators connect quarks and WIMPs. Precision electroweak measurements prevent these mediators to be SM gauge bosons. Different mono-X+MET channels test different DM mediator hypothesis: vector, axial-vector, scalar or pseudo-scalar. On the left of Figure 1 the Feynman diagram of a WIMP-pair-production, with an accompanying initial-state radiation gluon, is shown, where a $Z$ '-like particle has an axial-vector coupling $\mathrm{g}_{\chi}$ to dark matter particles and $\mathrm{g}_{q}$ to quarks.

DM mediators, if sufficiently massive, can also decay back to quarks, as shown on the right of Figure 1, leading to the signature of a bump in di-jet invariant mass. Consequently, searches for di-jet resonances could be interpreted in term of the production of a new DM mediator. In addition, under the assumptions of Simplified models, DM searches at the LHC can be directly compared to DM direct searches in underground experiments, then exploiting the complementarity between the two. This comparison is not possible in the framework of Effective Field Theory (EFT), because this schema does not include the effects of resonant enhancement or off-shell production due to the mediator propagator.
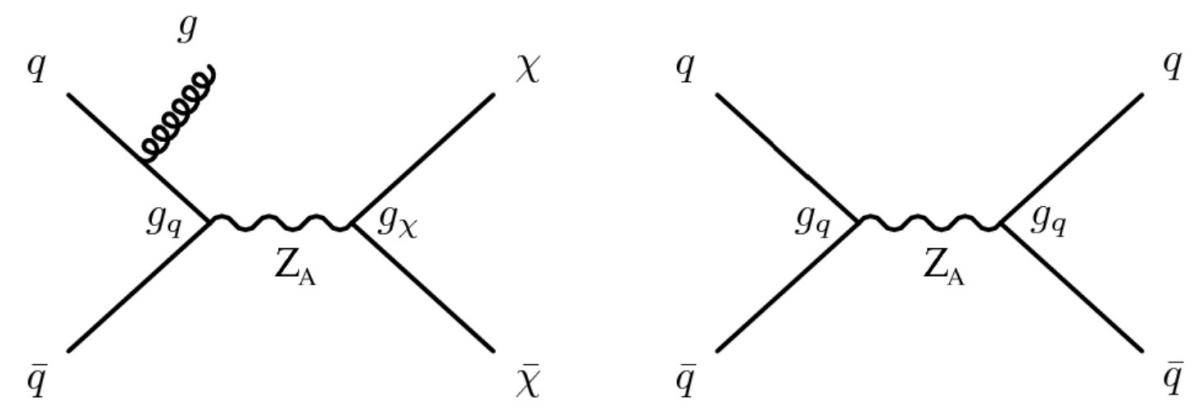

Figure 1: Production diagrams in the s-channel of a dark matter pair $\chi \chi$, recoiling against a gluon (left) and of a neutral dark matter mediator $Z_{A}$, decaying back to a pair of quarks (right). 


\section{Dark matter searches with missing energy: mono-X+MET}

The canonical mono-jet analysis has been performed in ATLAS [1] by requiring in the final state a high $\mathrm{p}_{T}>250 \mathrm{GeV}$ jet and a large missing transverse energy $\mathrm{E}_{T}^{\text {miss }}>250 \mathrm{GeV}$ [4]. Jets are reconstructed from energy deposits in the calorimeters using the anti- $k_{t}$ jet algorithm with a radius of 0.4. A separation in the azimuthal plane of $\Delta \phi\left(\right.$ jet, $\left.\vec{p}_{T}^{m i s s}\right)>0.4$, between the leading jet and the missing transverse momentum, reduces the multijet background, where jet energy mismeasurement can generate large $\mathrm{E}_{T}^{\text {miss }}$, keeping a high signal efficiency.

The multijet background amounts to only $0.3-0.4 \%$ of the total background in a few signal regions and it is negligible for most of the signal regions. It is determined from data, using the jet smearing method, which relies on the assumption that the $\mathrm{E}_{T}^{\text {miss }}$ of multijet events is dominated by fluctuations in the jet response of the detector, which can be measured in the data. The background is dominated by $\mathrm{Z}+$ jets, $\mathrm{W}+\mathrm{jets}$ and top-quark production processes with normalisation determined using control regions where the dark-matter signal is expected to be negligible.

Two signal regions are used: inclusive and exclusive regions with increasing requirements on $\mathrm{E}_{T}^{\text {miss }}$ (from $>250$ to $>1000 \mathrm{GeV}$ ). The first is optimised for model-independent searches and the second one for specific models interpretation. A simultaneous background-only likelihood fit to the $\mathrm{E}_{T}^{\text {miss }}$ distribution in the $\mathrm{W} / \mathrm{Z}+\mathrm{jets}$ and top control regions is performed to normalize and constrain the background estimates in the signal regions. It turns out a normalization factor of 1.27 for the $\mathrm{W} / \mathrm{Z}+$ jets background and 1.06 for the top-quark background.

In the left plot of Figure 2 the measured distributions of the $\mathrm{E}_{T}^{\text {miss }}$ for the described event selection is compared to the SM predictions determined by the global fit considering the exclusive signal regions. For illustration purposes, the distributions of different signal models are also included. Good agreement is observed between the data and the SM predictions in each case.
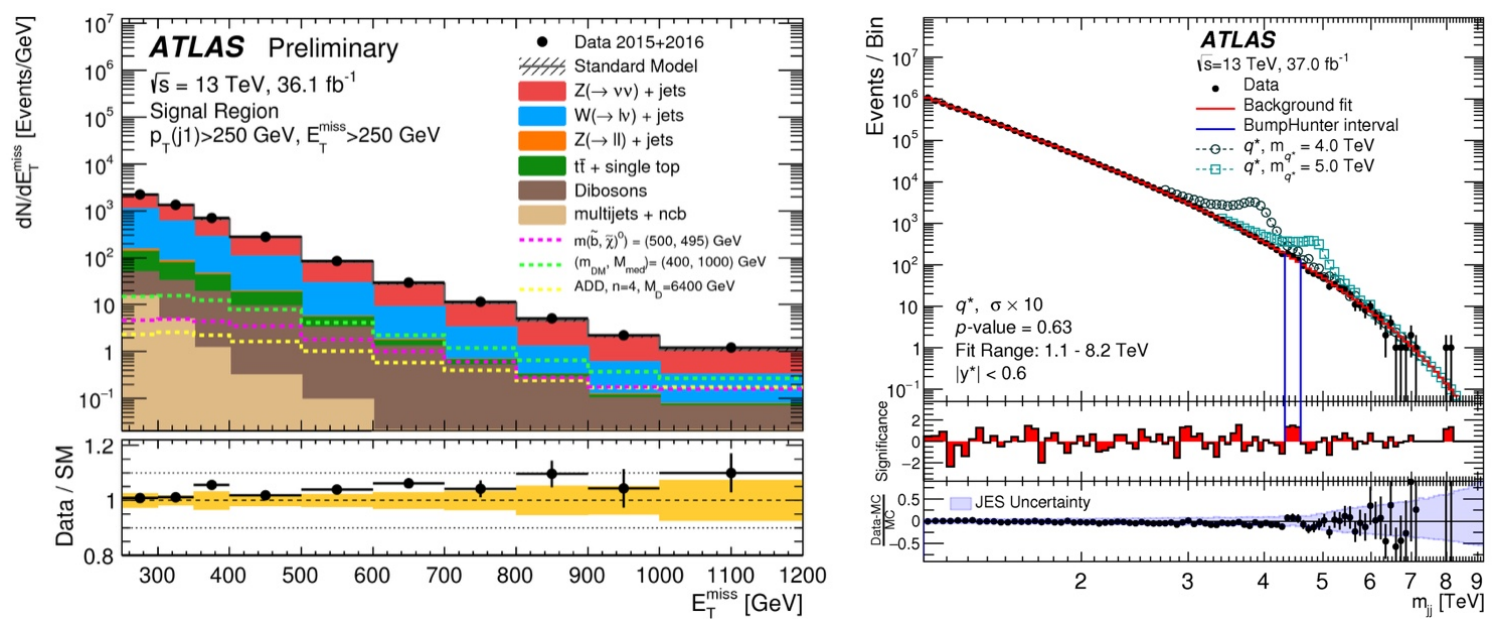

Figure 2: $\mathrm{E}_{T}^{\text {miss }}$ distribution with a Mono-jet (left) [4] and $\mathrm{m}_{j j}$ mass distribution for events with $|y *|<0.6$ (right) [5] compared to SM predictions. 


\section{Dark matter mediator searches: dijets resonances}

The di-jet analysis has always been used to search for new Z'-like resonances and contact interactions at a higher energy scale. Nowadays, it has received a renewed attention because DM mediator couples to ordinary matter and could decay back to ordinary matter.

The di-jet analysis performed by the ATLAS experiment looks for resonances in the $\mathrm{m}_{j j}$ spectrum and it is completely data-driven [5]. Collision events are recorded using a trigger that requires at least one jet reconstructed by the high-level trigger with a $\mathrm{p}_{T}$ greater than $380 \mathrm{GeV}$, the lowest- $\mathrm{p}_{T}$ unprescaled single-jet trigger. Events containing at least two jets are selected for offline analysis if the $\mathrm{p}_{T}$ of the leading (subleading) jet is greater than $440(60) \mathrm{GeV}$.

The di-jet invariant mass distribution is formed from the two leading jets in selected events to look for resonances. Background from QCD processes is reduced in nominal selection limiting the rapidity difference $\mathrm{y}^{*}=\left(\mathrm{y}_{1}-\mathrm{y}_{2}\right) / 2$ between the two leading jets at $|y *|<0.6$, where $\mathrm{y}=1 / 2 \ln \left[\left(\mathrm{E}+\mathrm{p}_{z}\right) /(\mathrm{E}-\right.$ $\mathrm{p}_{z}$ )], with $\mathrm{E}$ the energy and $\mathrm{p}_{z}$ the momentum component along the $\mathrm{z}$-axis of the jet.

The right plot of Figure 2 shows the measured $\mathrm{m}_{j j}$ distribution for events passing the selection, overlaid to background estimates and several example of signals. The background is derived from the sliding-window method: the invariant mass spectrum is constructed bin-by-bin by performing a likelihood fit to the data in each window and using the fit value at the center of the window.

The BumpHunter algorithm [6] quantifies the statistical significance of any localized excess in the $\mathrm{m}_{j j}$ distribution. The algorithm compares the binned $\mathrm{m}_{j j}$ distribution of the data to the fitted background estimate, considering contiguous mass intervals in all possible locations, from a width of two bins to a width of half of the distribution. The interval $[4326,4595] \mathrm{GeV}$, indicated by the two vertical lines in the right plot of Figure 2, is the most discrepant one in the signal region. The global significance of this outcome is 0.63 . Thus, there is no evidence of a localized contribution to the mass distribution from new phenomena.

For comparison the lower panel of the right plot of Figure 2 shows the relative differences between the data and the simulation of multijet production generated by Pythia 8.186 after being re-weighted to next-to-leading-order (NLO) with NLOJET++ and for electroweak effects.

\section{Exclusion plot and comparison with direct dark matter searches}

The interpretation of null results of searches for mono-X+MET and di-jet resonances can be projected onto the DM mass-mediator mass plane with assumptions on the couplings $\mathrm{g}_{\chi}$ and $\mathrm{g}_{q}$. In the left plot of Figure 3 the exclusion plot overlays results from different searches within the ATLAS experiment in the hypothesis of leptophobic axial-vector mediator. Vertical exclusion regions are produced by di-jet searches, because mediators decay back to mass-less quark, and triangular exclusion regions are due to mono-X+MET, because mediator decay to massive DM.

Collider and non-collider searches can be compared when interpreted in the framework of Simplified models. In fact, constraints on the DM mass - mediator mass plane are translated into upper limits on the DM nucleon scattering cross-section as a function of the mass of the DM particle. The right plot of Figure 3 overlays ATLAS results and PICO-60 [7] results of DM searches assuming a specific interaction structure and coupling scenarios. 

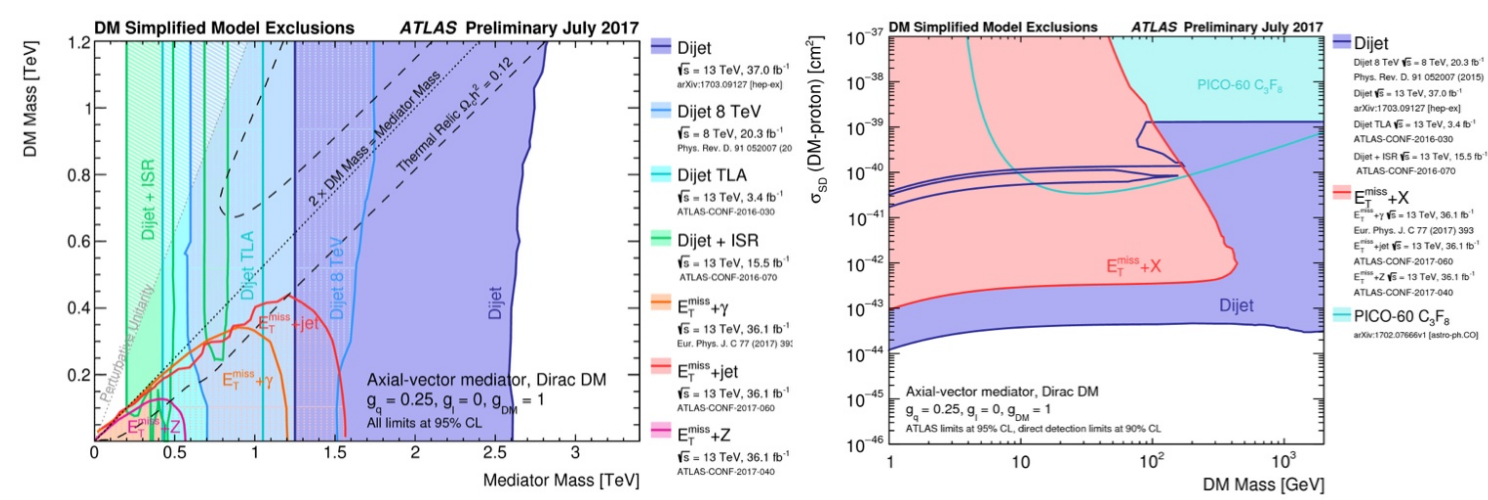

Figure 3: On the left plot the 95\% CL excluded regions in a DM mass-mediator mass plane from a selection of ATLAS dark matter searches using a leptophobic axial-vector mediator as described in [8]. On the right plot a comparison of the inferred limits on the spin-dependent dark matter-proton scattering cross section at $95 \%$ CL for ATLAS and at 90\% CL for the direct dark matter detection experiment PICO-60 based on superheated fluid. The plots are taken from [9].

\section{Conclusions}

The search for dark matter is an important part of the ATLAS physics program because the unprecedented energy reached by the LHC at CERN has allowed exploration of previously inaccessible kinematic regimes in the search of new phenomena. In this brief report, only the most general results of ATLAS concerning searches for dark matter and dark matter mediated interactions have been described. The theoretical framework of Simplified models provides an easy interpretation of the null results and a comparison with the constrains of direct dark matter searches in underground experiments. No evidence of physics beyond the Standard Model is observed so far by ATLAS, and limits are set on vector, axial-vector, and scalar coupling of dark matter mediators to ordinary fermions.

\section{References}

[1] ATLAS Collaboration, The ATLAS Experiment at the CERN Large Hadron Collider, 2008 JINST 3 S08003.

[2] G. Bertone, D. Hooper and J. Silk, Particle dark matter: Evidence, candidates and constraints, Phys. Rept. 405 (2005) 279, arXiv: hep-ph/0404175 [hep-ph].

[3] G.W. Angus, B. Famaey, H.S. Zhao, Can MOND take a bullet? Monthly Notices of the Royal Astronomical Society, 371, (2006) 138.

[4] ATLAS Collaboration, Search for dark matter and other new phenomena in events with an energetic jet and large missing transverse momentum using the ATLAS detector, ATLAS-CONF-2017-060, 2017, url: http://cds.cern.ch/record/2273876.

[5] ATLAS Collaboration, Search for new phenomena in dijet events using $37 \mathrm{fb}^{-1}$ of pp collision data collected at $\sqrt{s}=13 \mathrm{TeV}$ with the ATLAS detector, CERN-EP-2017-042, 2017, arXiv: 1703.09127 [hep-ex]. 
[6] G. Choudalakis, On hypothesis testing, trials factor, hypertests and the BumpHunter, 2011, arXiv: 1101.0390 [physics.data-an].

[7] C. Amole et al., Dark Matter Search Results from the PICO-60 $C_{3} F_{8}$ Bubble Chamber, Phys. Rev. Lett. 118, (2017) 251301.

[8] A. Albert et al., Recommendations of the LHC Dark Matter Working Group: Comparing LHC searches for heavy mediators of dark matter production in visible and invisible decay channels CERN- LPCC-2017-01, 2017, arXiv:1703.05703 [hep-ph].

[9] ATLAS Collaboration, Summary plots from the ATLAS Exotic physics group, url: https://atlas.web. cern.ch/Atlas/GROUPS/PHYSICS/CombinedSummaryPlots/EXOTICS/index.html. 